\title{
AKR1C3-activated Prodrug OBI-3424
}

National Cancer Institute

\section{Source}

National Cancer Institute. AKR1C3-activated Prodrug OBI-3424. NCI Thesaurus. Code C156414.

A small-molecule nitro-benzene, aldo-keto reductase 1C3 (AKR1C3)-activated prodrug of N,N'-bisethylenephosphoramidate, a DNA bis-alkylating agent, with potential antineoplastic activity. Upon intravenous administration, AKR1C3-activated prodrug OBI3424 is converted to its active form by AKR1C3, which is upregulated in certain tumor cell types while not expressed in normal healthy cells. The active metabolite selectively binds to and alkylates DNA in AKR1C3-overexpressing tumor cells, resulting in DNA base pair mismatching, interstrand crosslinking and inhibition of DNA repair and synthesis, cellcycle arrest, and apoptosis. As the expression of AKR1C3 is restricted to tumors, OBI3424 is selectively converted to its active metabolite in tumor cells only while its conversion in normal, healthy tissue is absent; this allows for an increased cytotoxic effect of the alkylating agent in tumor cells while decreasing its toxicity. 